\title{
Association between rates of caesarean section and maternal and neonatal mortality in the 21st century: a worldwide population-based ecological study with longitudinal data
}

\author{
J Ye, ${ }^{a, b}$ J Zhang, ${ }^{\text {a }}$ R Mikolajczyk, ${ }^{\text {c,d }}$ MR Torloni, ${ }^{\text {e,f }}$ AM Gülmezoglu, ${ }^{b}$ AP Betran ${ }^{b}$ \\ a Ministry of Education - Shanghai Key Laboratory of Children's Environmental Health, Xinhua Hospital, Shanghai Jiao Tong University \\ School of Medicine, Shanghai, China ${ }^{\mathrm{b}}$ Development and Research Training in Human Reproduction, Department of Reproductive Health \\ and Research, UNDP, UNFPA, UNICEF, WHO, World Bank Special Programme of Research, World Health Organization, Geneva, \\ Switzerland ${ }^{\mathrm{c}}$ ESME - Epidemiological and Statistical Methods Research Group, Helmholtz Centre for Infection Research, Braunschweig, \\ Germany ${ }^{\mathrm{d}}$ Hannover Medical School, Hannover, Germany ${ }^{\mathrm{e}}$ Brazilian Cochrane Center, São Paulo School of Medicine, São Paulo Federal \\ University, São Paulo, Brazil ${ }^{\mathrm{f}}$ Department of Obstetrics, São Paulo School of Medicine, São Paulo Federal University, São Paulo, Brazil \\ Correspondence: Dr J Zhang, Xinhua Hospital, Shanghai Jiao Tong University School of Medicine, 1665 Kong Jiang Road, Shanghai 200092, \\ China. Email junjimzhang@gmail.com
}

Accepted 7 July 2015. Published Online 24 August 2015

Objective Caesarean section was initially performed to save the lives of the mother and/or her baby. Caesarean section rates have risen substantially worldwide over the past decades. In this study, we set out to compile all available caesarean section rates worldwide at the country level, and to identify the appropriate caesarean section rate at the population level associated with the minimal maternal and neonatal mortality.

Design Ecological study using longitudinal data.

Setting Worldwide country-level data.

Population A total of 159 countries were included in the analyses, representing $98.0 \%$ of global live births (2005).

Methods Nationally representative caesarean section rates from 2000 to 2012 were compiled. We assessed the relationship between caesarean section rates and mortality outcomes, adjusting for socio-economic development by means of human development index (HDI) using fractional polynomial regression models.

Main outcome measures Maternal mortality ratio and neonatal mortality rate.
Results Most countries have experienced increases in caesarean section rate during the study period. In the unadjusted analysis, there was a negative association between caesarean section rates and mortality outcomes for low caesarean section rates, especially among the least developed countries. After adjusting for HDI, this effect was much smaller and was only observed below a caesarean section rate of $5-10 \%$. No important association between the caesarean section rate and maternal and neonatal mortality was observed when the caesarean section rate exceeded $10 \%$.

Conclusions Although caesarean section is an effective intervention to save maternal and infant lives, based on the available ecological evidence, caesarean section rates higher than around $10 \%$ at the population level are not associated with decreases in maternal and neonatal mortality rates, and thus may not be necessary to achieve the lowest maternal and neonatal mortality.

Keywords Caesarean section rate, maternal mortality, neonatal mortality.

Tweetable abstract The caesarean section rate of around 10\% may be the optimal rate to achieve the lowest mortality.

Please cite this paper as: Ye J, Zhang J, Mikolajczyk R, Torloni MR, Gülmezoglu AM, Betran AP. Association between rates of caesarean section and maternal and neonatal mortality in the 21st century: a worldwide population-based ecological study with longitudinal data. BJOG 2016;123:745-753.

\section{Introduction}

Caesarean section rates have risen substantially worldwide since the 1980s. ${ }^{1-4}$ In some countries the rate has increased several-fold since 1985, when the World Health Organization (WHO) suggested a caesarean section rate of $10-15 \%$ as the upper limit at the population level. ${ }^{5}$ Although some less developed countries, mostly in Africa, still have the rate below $10 \%$, the majority of the countries have surpassed this recommendation and some have exceeded it by a wide margin. ${ }^{1,2,4}$ The reasons for such dramatic increases are multifactorial, including medical as well as non-medical 
reasons. ${ }^{3,6,7}$ It has been suggested that non-medical factors, such as social, cultural, or unequal accessibility to health services, as well as clinical practice patterns/styles, might have been major contributors to the wide variation in caesarean section rates across different countries. ${ }^{1,2,6-10}$ On the other hand, we can presume that the rates of medically necessary caesarean section associated with the lowest maternal and neonatal mortality should vary less among countries, if the same indications for caesarean section had been applied across all nations. ${ }^{4,11}$ What, then, should be the appropriate caesarean section rate?

In an attempt to identify such a caesarean section rate, several ecological studies have analysed the association between mode of delivery and maternal, neonatal and infant mortality. ${ }^{2,4,12-18}$ They used different statistical methods for the analysis (e.g. piecewise regression, exponential models, quadratic models, locally weighted scattered plot smoothing, and fractional polynomial regression models), and found a nonlinear relationship between caesarean section and maternal, neonatal, and infant mortality. ${ }^{2,4,13,17,18}$ It was observed that high caesarean section rates were associated with lower maternal and infant mortality till a specific point, above which caesarean section rates were not significantly associated with these outcomes. This inflection point was considered as a necessary caesarean section rate from a medical viewpoint to minimise mortality; however, the results and interpretations of these studies were limited by the cross-sectional nature of the ecological data. Moreover, most of these studies did not control for socio-economic conditions, which can have a great impact on both the availability of caesarean section and maternal and perinatal mortality, and thus confound the association between caesarean section rates and adverse outcomes. ${ }^{2,12,13,16-18}$ We therefore set out to compile available caesarean section rates worldwide at the country level, and assess the association between mode of delivery and maternal and neonatal mortality using a longitudinal approach and adjusting for socio-economic development.

\section{Methods}

\section{Definitions and data sources}

The rate of caesarean section is defined as a percentage calculated by dividing the number of caesarean deliveries over the total number of live births in a period of time, normally 1 year. We compiled all nationally representative data on caesarean section rates available since 1980 . The sources of data for the caesarean section rate for each country are presented in Table S1. In brief, information on caesarean section rates was obtained from several data sources.

1 Caesarean section rates reported in the Demographic and Health Surveys (DHS) programme (www.measuredhs.com) or Multiple Indicator Cluster Surveys (MICS, mics.unicef.org). These surveys represent the lar- gest worldwide effort to obtain demographic and health data from nationally representative household surveys in developing countries. DHS are conducted about every 5 years since 1984 . MICS has been carried out in more than 100 countries every 5 years since 1995. The technical teams developing and supporting MICS and DHS are in close collaboration. Data from these surveys are highly comparable. $^{19}$

2 The European Health for All Database (www.who.dk), maintained by the WHO European Regional Office, which includes basic demographic, socio-economic and health-related indicators that are obtained from routine national registries.

3 Caesarean section rates reported in routine statistical surveillance system reports or national surveys from government health offices. For example, in the USA, Australia, and New Zealand, information on maternal health indicators was regularly updated on government websites. Data are obtained from routine national registries.

4 For the few countries that do not routinely publish caesarean section rates, we searched journal articles in MEDLINE, EMBASE, and reference lists to collect nationally representative data (e.g. caesarean section rates based on national surveys officially reported by National Bureau of Statistics or Ministry of Health).

Maternal and neonatal mortality data for all countries were obtained from the World Health Statistics Report. The World Health Statistics Report is published yearly by the WHO and presents a series of health-related indicators for all member states (www.who.int/gho/publications/ world_health_statistics/en/). The maternal mortality ratio (MMR; maternal deaths per 100000 live births) and the neonatal mortality rate (NMR; neonatal death within 28 days of birth per 1000 live births) were the outcome indicators used in this analysis. Country, regional, and global estimates are produced by WHO in an inter-agency coordinated effort, and are used for international monitoring efforts and the Millennium Development Goals.

The information on country-level Human Development Index (HDI) was obtained from the Human Development Reports published by the United Nations Development Programme (hdr.undp.org/en/data). HDI measures average achievements in a country in three basic dimensions of human development: a long and healthy life; access to knowledge; and a decent standard of living (gross national income, GNI, per capita). It evaluates development not only by economic advances but also by improvements in human wellbeing. It provides a proxy for important confounding factors in this analysis, such as health status of pregnant women, and the availability and actual provision of healthcare services. As an index of social welfare, some authors have used HDI data to measure the impact of economic policies on quality of 
life. $^{20}$ We assumed that the HDI could provide a more comprehensive proxy for confounding factors, such as availability and the actual provision of healthcare services, in this analysis than its constituents: life expectancy, education index, and GNI/gross domestic product (GDP).

We compiled all available nationally representative caesarean section rates from 1980 to 2012; however, because of the incompleteness of information on caesarean section rate, mortality outcomes, and HDI, especially in developing countries before 2000, we restricted our analyses to the time period between 2000 and 2012. These data also represent the contemporary situation of caesarean section rates and mortality better. Countries, year, and caesarean section rates of the first and last observation, the number of caesarean section rates available, and the original source of the data for the latest year are presented in Table S1.

\section{Statistical methods}

In our study, most countries have provided the caesarean section rate, and maternal and neonatal mortality, more than once; many developed countries reported a series of annual data points. Such data offered us an opportunity to carry out an ecological study using a longitudinal approach with a repeated-measures analysis. Each country represented one unit of analysis. Country-level caesarean section rates, socio-economic conditions, and mortality outcomes were repeated measurements of each country. Of the least and the less developed countries, 76 and $61 \%$ had less than three data points of caesarean section rates available, respectively. Of the less and the more developed countries, 20 and $81 \%$ had more than ten data points, respectively. None of the least developed countries had more than six data points available.

We described the changes of caesarean section rates, mortality outcomes, and HDI over time. The number of annual live births of each country was used as weighting to calculate the average global/regional caesarean section rate, maternal mortality ratio, and neonatal mortality rate. The regional coverage was calculated as the proportion of total regional live births for which nationally representative data on caesarean section were available. In the descriptive analysis, if the caesarean section rate in 2012 was not available, the newest data after 2008 was presented instead. Caesarean section rate and HDI were not always available, whereas the mortality data were available for each country. We therefore performed a linear interpolation of caesarean section rates and HDI for each country. The proportions of interpolated caesarean section rates during the study period (2000-2012) in the least, less, and more developed countries were $85.9,68.8$, and $22.4 \%$, respectively. Missing values after the last observation available were not imputed in the analysis of association between caesarean section rates and mortality rates. Observations with missing values of caesarean section rate, mortality rate, or HDI were excluded from the regression analysis. Sensitivity analyses were performed using the original data set (without interpolation) to detect potential impact of imputation on the relationship between caesarean section rates and mortality outcomes.

Two-level mixed models were used to describe the relationship between caesarean section rate and maternal and neonatal mortality rates, with and without adjusting for HDI, in a longitudinal analysis. Caesarean section rates, socio-economic conditions, and mortality outcomes nested within countries were repeated measurements at level 1. Each country represented one unit at level 2. Because maternal death was a very rare outcome, the maternal mortality ratio was transformed to a log scale for the analysis of its association with caesarean section rates, whereas a linear model was used for the association between caesarean section rates and neonatal mortality.

Fractional polynomial regression models were used to fit the nonlinear relationship between caesarean section rates and mortality outcomes. Fractional polynomial regression is an extension of polynomial regression models. The power term of the independent variable can take integer or non-integer values, so that the fractional polynomial regression model provided more flexible and better fitting than the other methods. The powers are chosen from a predefined set of values $(-2,-1,-0.5,0,0.5,1,2,3)$. The best-fitting model is defined as the one that maximises the likelihood (minimises the deviance). The details of the methods were described elsewhere. ${ }^{21,22}$ Second-degree fractional polynomials transformation was performed for caesarean section rates to obtain more flexibility. HDI was standardised and entered as a linear effect in the adjusted models. The best model (with minimum -2 log-likelihood) was chosen from all alternative second-degree fractional polynomial models. ${ }^{21,22}$ Deviance residuals plotted against caesarean section rates showed no non-random trends or patterns for the selected models. Because it is difficult to interpret the meaning of coefficients of the fractional polynomial transformation of caesarean section rates, we presented the results of the second-degree fractional polynomial models for mortality rates as a function of caesarean section rates in Table S2 and the predicted mortality rates in Table S3.

We conducted the analyses for all countries first and then performed stratified analyses within subgroups of least, less, and more developed countries according to UN classification. $^{23}$ All analyses were performed using SAS 9.2 (SAS Institute Inc., Cary, NC, USA). 


\section{Results}

A total of 159 countries reporting at least one caesarean section rate during the study period were included in the analyses, representing 98.0\% of global live births in 2005 . Among them, 41, 75, and 43 were least, less, and more developed countries, respectively. The coverage of our data in these categories was $91.8,99.7$, and $99.2 \%$, respectively.

Table 1 shows the changes of caesarean section rate, mortality rates, and HDI in the 13-year period. A total of 120 countries had records of caesarean section rate in 2000, representing $90.1 \%$ global live births. The coverage of live births in the least, less, and more developed countries was $74.1,93.2$, and $98.9 \%$, respectively. The average world caesarean section rate was $12.0 \%$, with the highest average recorded in the more developed countries (19.5\%) and the lowest rate recorded in the least developed countries (2.0\%).

A total of 132 countries were included in the analysis of 2012, representing $74.3 \%$ of global live births. The coverage of live births in the least, less, and more developed categories was $85.4,67.3$, and 99.2\%, respectively. A total of 13 countries included in the description of 2000 had no records in 2012 (or after 2008) and, therefore, were not included in the analysis of 2012. Some of these counties had a large number of live births (e.g. India), which may lead to dramatically decreased coverage. The average global caesarean section rate increased to $15.5 \%$, with a range from $1.4 \%$ in Niger to $55.6 \%$ in Brazil. In the least devel- oped category, caesarean section rates increased slightly to an average rate of 5.2\%. All more developed countries had caesarean section rates higher than 15\%, except for Bosnia and Herzegovina (13.9\%) and Finland (14.7\%). Less developed countries provided more diverse data with caesarean section rates ranging from $1.7 \%$ in Timor-Leste to $55.6 \%$ in Brazil. Although more developed countries had the largest absolute increase in caesarean section rates $(7.4 \%)$, the least developed countries showed the greatest relative changes of caesarean section rate (caesarean section rates increased $160 \%$ compared with the baseline) during the study period.

In 2000, maternal mortality in the least, less, and more developed categories was 607.0, 288.8 and 17.5 per 100000 live births, respectively (Table 1). By 2012, maternal mortality had declined sharply to 432.3 and 175.6 per 100000 in the least and the less developed categories, respectively, but only slightly in more developed countries, to 16.2 per 100 000. The pattern of decrease in neonatal mortality rate was similar to that of maternal mortality.

In all development categories, maternal and neonatal mortality rates decreased sharply with increasing caesarean section rates when caesarean section rates were below 5-10\% (Figures 1-3). The curves became flatter when caesarean section rates exceeded $10 \%$. The association between caesarean section rates and mortality rates was even weaker after adjustment for HDI. The least developed countries had much higher maternal and neonatal mortality than the less and the more developed countries with the same cae-

Table 1. Caesarean section rates, mortality outcomes, and human development index by region, 2000-2012*

\begin{tabular}{|c|c|c|c|c|}
\hline & World total & Least developed & Less developed & More developed \\
\hline \multicolumn{5}{|c|}{ Caesarean section rate, \% (range) } \\
\hline 2000 & $12.0(0.5-38.0)$ & $2.0(0.5-6.4)$ & $13.1(1.5-38.0)$ & $19.5(7.5-33.3)$ \\
\hline 2012 & $15.5(1.4-55.6)$ & $5.2(1.4-17.9)$ & $16.9(1.7-55.6)$ & $26.9(13.9-38.1)$ \\
\hline Relative change ${ }^{\star *}$ & 1.3 & 2.6 & 1.3 & 1.4 \\
\hline \multicolumn{5}{|c|}{ Maternal mortality, per 100000 live births (range) } \\
\hline 2000 & $309.8(4.0-1100.0)$ & $607.0(340.0-1100.0)$ & $288.8(9.0-970.0)$ & $17.5(4.0-57.0)$ \\
\hline $2010 * * *$ & $208.7(2.0-1100.0)$ & $432.3(79.0-1100.0)$ & $175.6(7.0-690.0)$ & $16.2(2.0-41.0)$ \\
\hline \multicolumn{5}{|c|}{ Neonatal mortality, per 1000 live births (range) } \\
\hline 2000 & $28.1(1.7-54.0)$ & $39.4(26.9-54.0)$ & $29.1(3.5-48.5)$ & $5.0(1.7-13.5)$ \\
\hline $2012 * * *$ & $20.6(0.8-49.5)$ & $29.4(18.2-49.5)$ & $20.7(1.6-42.2)$ & $3.5(0.8-8.6)$ \\
\hline \multicolumn{5}{|c|}{ Mean human development index (range) } \\
\hline 2000 & $0.63(0.23-0.91)$ & $0.35(0.23-0.44)$ & $0.61(0.37-0.86)$ & $0.81(0.59-0.91)$ \\
\hline $2011 * * *$ & $0.65(0.29-0.94)$ & $0.41(0.29-0.57)$ & $0.66(0.38-0.90)$ & $0.84(0.65-0.94)$ \\
\hline \multicolumn{5}{|c|}{ Coverage of live births } \\
\hline 2000 & 90.1 & 74.1 & 93.2 & 98.9 \\
\hline 2012 & 74.3 & 85.4 & 67.3 & 99.2 \\
\hline
\end{tabular}


sarean section rates. The predicted neonatal mortality rates of the adjusted model in the least developed category declined significantly from 41.8 (95\% confidence interval, 95\% CI 39.5-44.0) to 36.4 (95\% CI 33.9-38.9), per 1000 live births, with an increasing caesarean section rate from 1 to $10 \%$. The corresponding maternal mortality rates decreased from 681.9 (95\% CI 592.4-785.1) to 480.1 (95\% CI 415.4-554.9) per 100000 live births. In less developed countries, the predicted neonatal mortality rates decreased from 23.3 (95\% CI 21.3-25.2) to 20.3 (95\% CI 19.1-21.5), per 1000 live births, when caesarean section rates increased from 1 to $10 \%$, whereas maternal mortality rates decreased from 183.1 (95\% CI 70.8-473.3) to 118.6 (95\% CI 101.9-138.0), per 1000 live births.

All more developed countries had a caesarean section rate higher than $10 \%$, whereas only two of the least developed countries had caesarean section rates higher than $10 \%$ (Bhutan, 12.4\%; Bangladesh, 17.9\%). In the less and the more developed countries, neonatal mortality decreased monotonically (but not significantly for every 5\% increase in caesarean section rate). In contrast, maternal mortality increased slightly when caesarean section rates were higher than 10\% (Table S3). Models and predicted values of these curves were presented in Tables S2 and S3.

We conducted sensitivity analyses using the original data (without imputation) to detect the effects of interpolation on the results. The trends and cut points of curves fitted to the models were essentially unchanged (Figures S1-S3).

\section{Discussion}

\section{Main findings}

The rates of caesarean section have increased in most countries in the world over the last 12 years. More developed countries had higher baselines and greater increases in cae- sarean section rates than least and less developed countries in absolute terms; however, maternal and neonatal mortality rates in these countries changed only slightly during the corresponding period. On the other hand, the least developed countries showed the most relative increase in caesarean section rates. There was a substantial decrease in maternal and neonatal mortality with a small absolute increase of caesarean section rates in the least developed countries. The significant and negative relationship between caesarean section rates and mortality was only found when the caesarean section rate was below 5-10\%. After adjusting HDI the association was less pronounced, i.e. the crude association appears to be mainly explained by socio-economic conditions. In addition, our analysis suggests that a caesarean section rate higher than around $10 \%$ at a population level may not be significantly correlated with maternal and neonatal mortality.

\section{Strengths and limitations}

Determining what is the appropriate caesarean section rate at the population level is a very challenging task, especially when it is not currently feasible to obtain perfect data worldwide. Socio-economic conditions, cultural, and health system factors have a large impact on both caesarean section rates and maternal and neonatal mortality rates, and, therefore, are significant confounding factors. ${ }^{4,18,24}$ To minimise their confounding effects, we conducted a longitudinal data analysis with a mixed model to adjust for HDI. In contrast to a cross-sectional ecological study that compared data from different countries, our analysis was based on changes observed within each country. As such, the model controlled automatically for characteristics of the country that did not change over time. Adjusting for HDI in each year allowed us to control for changing socioeconomic conditions within the country (to the degree to
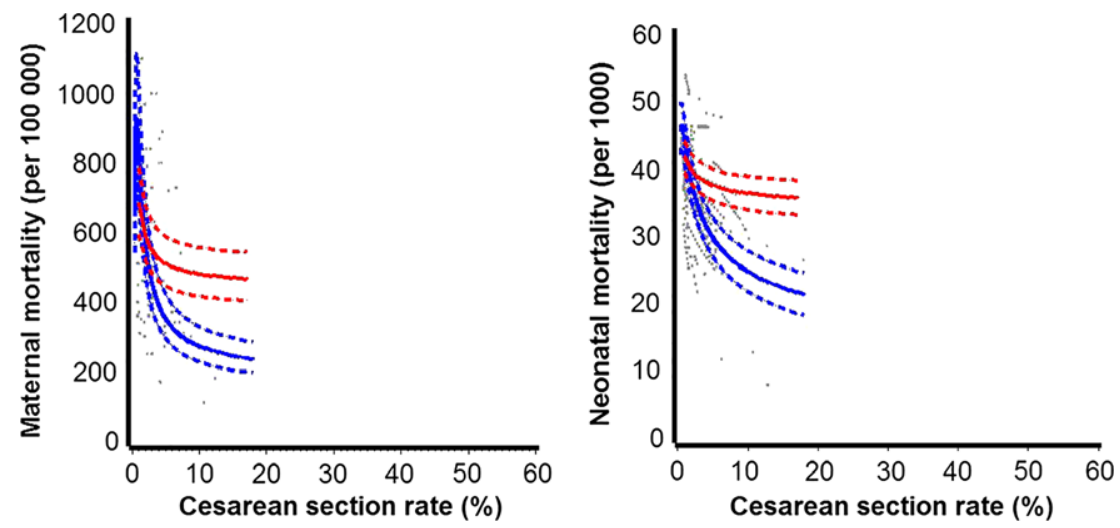

Figure 1. Relationship between caesarean section rates and maternal mortality and neonatal mortality, with and without adjusting for human development index (HDI), for least developed countries. Scatter plots and fractional polynomial regression model (estimates and $95 \%$ confidence intervals): blue curves, unadjusted models; red curves, adjusted models; solid curves, estimates; dashed curves, 95\% Cls. (HDI standardised, mean HDI used to fit the curves of the adjusted model). 
Ye et al.
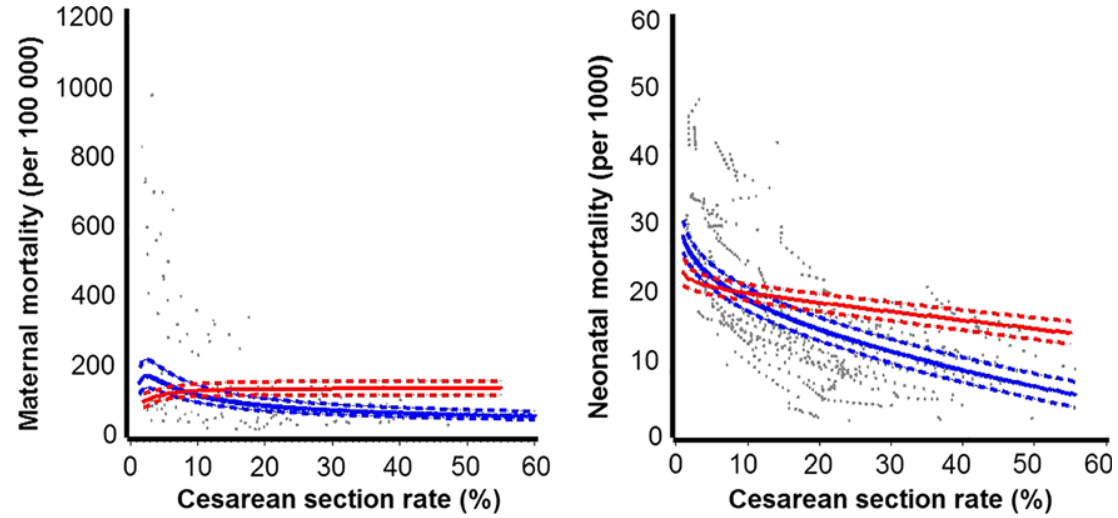

Figure 2. Relationship between caesarean section rates and maternal mortality and neonatal mortality, with and without adjusting for human development index (HDI), for less developed countries. Scatter plots and fractional polynomial regression model (estimates and $95 \%$ confidence interval): blue curves, unadjusted models; red curves, adjusted models; solid curves, estimates; dashed curves, 95\% CI. (HDI standardised, mean HDI used to fit the curves of the adjusted model).
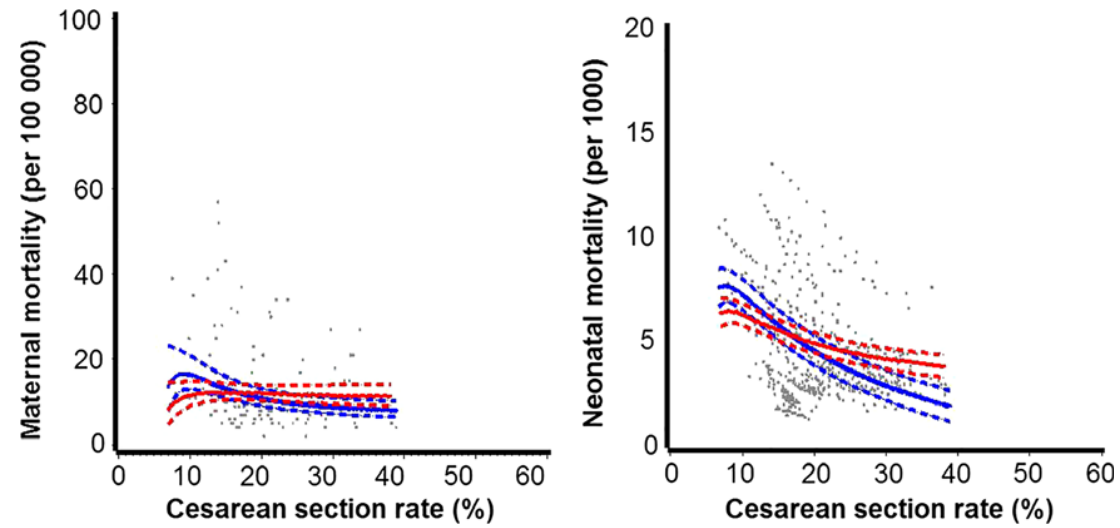

Figure 3. Relationship between caesarean section rates and maternal mortality and neonatal mortality, with and without adjusting for human development index (HDI), for more developed countries. Scatter plots and fractional polynomial regression model (estimates and $95 \%$ confidence interval): blue curves, unadjusted models; red curves, adjusted models; solid curves, estimates; dashed curves, 95\% CI. (HDI standardised, mean HDI used to fit the curves of the adjusted model).

which they were reflected in HDI development over time). Furthermore, we used the fractional polynomial regression model to fit the association between caesarean section rate and mortality outcomes. The power term of the independent variable can take integer or non-integer values, which provided more flexible and better fitting than the other methods. ${ }^{21}$

Our study has several limitations. Ecological studies may establish an association but not necessarily causality. The validity of an ecological association depends on the ability to control for differences among countries in the joint distribution of confounding factors. The correlation of confounding factors at the population level is usually higher than that at the individual level, and more difficult to disentangle. ${ }^{25,26}$ In the current study, socio-economic conditions, represented by the HDI, were adjusted when we modelled the relationship between caesarean section rates and mortality; however, residual confounding as a result of socio-economic conditions may still exist. Moreover, for all countries, the last available values of HDI were in 2011. Thus, caesarean section rates and mortality rates in 2012 were not used in the adjusted models.

In addition, stillbirth was not used as an outcome in this study because of a lack of longitudinal information on this outcome from most countries. Furthermore, it may be argued that maternal and neonatal deaths are extreme and rare outcomes. They may still be good indicators for less developed countries, but not sensitive enough for countries with low mortality, in which health professionals are more concerned with morbidity and quality of life as outcome indicators. ${ }^{27-29}$ Therefore, the caesarean section rate threshold identified in this analysis is not applicable to outcomes other than mortality. It is likely that to prevent such outcomes as near miss, maternal and perinatal morbidity, 
and long-term adverse outcomes, a higher threshold of caesarean section rate may be needed. It would be important in the future to assess the association of caesarean section rates with short- and long-term maternal and perinatal morbidity at the population level.

\section{Interpretation}

Over the past three decades the WHO-proposed caesarean section rate of $10-15 \%$ was used as a threshold, ${ }^{5}$ despite the lack of concrete evidence to support this statement. Several ecological studies have analysed the association between the mode of delivery and maternal, neonatal, and infant mortality, and identified an inflection point below which there was a strong association between caesarean section rates and mortality outcomes. ${ }^{2,4,12-18}$ A systematic review of ecological studies concluded that this point ranged from 9 to $16 \%$, and at caesarean section rates above this threshold, there was no longer an association between caesarean section rates and maternal or infant mortality. ${ }^{30}$ Among the eight studies found, only two previous ecological studies controlled for socio-economic conditions. One of them found a negative association between caesarean section rates and maternal mortality, even after adjusting for gross national income, proportion of deliveries with skilled attendance, and proportion of literate population in low-income countries. ${ }^{14}$ The observed negative association between caesarean section rates and neonatal mortality became statistically non-significant after adjusting for socio-economic conditions. But the authors did not demonstrate an inflection point of caesarean section rate based on adjusted models. ${ }^{14}$

The second study supported the WHO's reference, and suggested a caesarean section rate at $10-15 \%$ based on its association with mortality outcomes after adjusting for GDP and HDI. ${ }^{4}$ Our analysis demonstrated a substantially less pronounced association after adjusting for socio-economic development, and suggested an even lower threshold for a significant association between caesarean section rates and maternal and neonatal mortality at the population level.

The difference in the inflection points suggested by these studies may result from the different covariates used in the analyses. In previous studies GNI and proportion of literate population, or GDP and HDI, were adjusted. ${ }^{4,14}$ GDP, GNI, and proportion of literate population are all components of the HDI. We assumed that HDI could provide a more comprehensive proxy for confounding factors such as availability and actual provision of healthcare services, which should be adjusted when we study the relationship between caesarean section rate and mortality. Our study found that the association between caesarean section rates and mortality was strongly influenced by socio-economic status. Therefore, socio-economic differences may be an important factor that could explain most of the crude association between caesarean section rate and mortality outcomes. After adjusting for HDI, the significant and negative association between caesarean section rate and mortality was observed when the caesarean section rate was lower than around $10 \%$. The inflection point appears lower than the numbers reported by other investigators. ${ }^{4,13,16-18}$

Nonetheless, we need to caution against the potential misinterpretation of these results. Caesarean section percentages and ranges obtained from this analysis are an approximation not an 'exact' number for the optimal rate of caesarean section. In addition, the interpretation and implications of these findings in particular countries warrant specific assessment. For example, in countries with high or very high caesarean section rates, the goal of reducing their caesarean section rate to $10 \%$ may not be safe or advisable over a span of just a few years. On the other hand, in countries with very low caesarean section rates, and in settings where there are not enough skilled health professionals, and/or equipment or infrastructure, when trying to increase caesarean section rates at the population level, the focus needs to be on ensuring the safe provision of caesarean sections and not just on increasing numbers.

\section{Conclusion}

Pending potential analyses with relevant morbidity outcomes, our ecological study suggests that, at the population level, caesarean section rates higher than around 10\% are not associated with substantial decreases in maternal and neonatal mortality after controlling for socio-economic conditions. Based on the available ecological evidence, higher caesarean section rates may not be necessary to achieve the lowest maternal and neonatal mortality rates at the population level.

\section{Disclosure of interests}

None declared. Completed disclosure of interests form available to view online as supporting information.

\section{Contribution to authorship}

JZ and APB conceived this study. JY analysed the data and drafted the article. RM and JZ supervised the data analysis. JY, JZ, RM, MRT, AMG, and APB provided critical feedback on the interpretation of the results and helped with the writing of the article.

\section{Details of ethics approval}

Country level data were obtained from public sources, rendering ethics approval unnecessary.

\section{Funding}

Funding for this project was provided by a grant from UNDP, UNFPA, UNICEF, WHO, World Bank Special Programme of Research, Development and Research 
Ye et al.

Training in Human Reproduction, Department of Reproductive Health and Research, World Health Organization, Geneva, Switzerland. The funding agency had no role in: the design and conduct of the study; the collection, management, analysis, and interpretation of the data; the preparation, review, or approval of the article; and the decision to submit the article for publication.

\section{Acknowledgements}

The authors are grateful to Dr Cinthia Stanton, Catherine Spong and Mr Daniel Wojdyla, who provided critical comments on data analysis and interpretation, and provided feedback on the draft article.

\section{Supporting Information}

Additional Supporting Information may be found in the online version of this article:

Figure S1. Relationship between caesarean section rates and maternal mortality and neonatal mortality, with and without adjusting for human development index (HDI), for the least developed countries, using original data without imputation.

Figure S2. Relationship between caesarean section rates and maternal mortality and neonatal mortality, with and without adjusting for human development index (HDI), for less developed countries, using original data without imputation.

Figure S3. Relationship between caesarean section rates and maternal mortality and neonatal mortality, with and without adjusting for human development index (HDI), for more developed countries using original data without imputation.

Table S1. Caesarean section rates of the first and last observation, the number of caesarean section rates available, and the original source, by country.

Table S2. Fractional polynomial regression models fitted for the relationship between caesarean section rates and mortality outcomes.

Table S3. Predicted mortality rates based on the fixed effects of mixed models fitted to the relationship between caesarean section rates and mortality outcomes.

\section{References}

1 Gibbons L, Belizán JM, Lauer JA, Betrán AP, Merialdi M, Althabe F. The Global Numbers and Costs of Additionally Needed and Unnecessary Caesarean Sections Performed per Year: Overuse as a Barrier to Universal Coverage. World Health Report (2010) Background Paper, No 30.

2 Betran AP, Merialdi M, Lauer JA, Bing-Shun W, Thomas J, Van Look $P$, et al. Rates of caesarean section: analysis of global, regional and national estimates. Paediatr Perinat Epidemiol 2007:21:98-113
3 Robson MS. Can we reduce the caesarean section rate? Best Pract Res Clin Obstet Gynaecol 2001;15:179-94.

4 Ye J, Betran AP, Guerrero Vela M, Souza JP, Zhang J. Searching for the optimal rate of medically necessary cesarean delivery. Birth (Berkeley, Calif) 2014:41:237-44.

5 Appropriate technology for birth. Lancet 1985;2:436-7.

6 Gibbons L, Belizan JM, Lauer JA, Betran AP, Merialdi M, Althabe F. Inequities in the use of cesarean section deliveries in the world. Am J Obstet Gynecol 2012;206:331.e1-19.

7 Zhang J, Troendle J, Reddy UM, Laughon SK, Branch DW, Burkman $\mathrm{R}$, et al. Contemporary cesarean delivery practice in the United States. Am J Obstet Gynecol 2010;203:326.e1-.e10.

8 Fioretti B, Reiter M, Betran A, Torloni M. Googling caesarean section: a survey on the quality of the information available on the Internet. BJOG 2015;122:731-9.

9 Torloni MR, Betran AP, Montilla P, Scolaro E, Seuc A, Mazzoni A, et al. Do Italian women prefer cesarean section? Results from a survey on mode of delivery preferences. BMC Pregnancy Childbirth 2013;13:78.

10 Torloni MR, Campos Mansilla B, Merialdi M, Betran AP. What do popular Spanish women's magazines say about caesarean section? A 21-year survey. BJOG 2014;121:548-55.

11 Joffe M, Chapple J, Paterson C, Beard RW. What is the optimal caesarean section rate? An outcome based study of existing variation. J Epidemiol Community Health 1994:48:406-11.

12 Jurdi R, Khawaja M. Caesarean section rates in the Arab region: a cross-national study. Health Policy Plan 2004;19:101-10.

13 McClure EM, Goldenberg RL, Bann CM. Maternal mortality, stillbirth and measures of obstetric care in developing and developed countries. Int J Gynaecol Obstet 2007;96:139-46.

14 Althabe F, Sosa C, Belizan JM, Gibbons L, Jacquerioz F, Bergel E. Cesarean section rates and maternal and neonatal mortality in low-, medium-, and high-income countries: an ecological study. Birth (Berkeley, Calif) 2006;33:270-7.

15 Belizan JM, Althabe F, Barros FC, Alexander S. Rates and implications of caesarean sections in Latin America: ecological study. BMJ (Clinical Research ed) 1999;319:1397-400.

16 Silva AA, Silva LM, Barbieri MA, Bettiol H, Carvalho LM, Ribeiro VS, et al. The epidemiologic paradox of low birth weight in Brazil. Rev Saude Publica 2010;44:767-75.

17 Volpe FM. Correlation of Cesarean rates to maternal and infant mortality rates: an ecologic study of official international data.. Rev Panam Salud Publica 2011;29:303-8.

18 Zizza A, Tinelli A, Malvasi A, Barbone E, Stark M, De Donno A, et al. Caesarean section in the world: a new ecological approach. J Prev Med Hyg 2011;52:161-73.

19 Hancioglu A, Arnold F. Measuring coverage in $\mathrm{MNCH}$ : tracking progress in health for women and children using DHS and MICS household surveys. PLoS Med 2013;10:e1001391.

20 Davies A, Quinlivan G. A panel data analysis of the impact of trade on human development. J Socio-Econ 2006 Oct;35(5):868-876.

21 Royston $P$, Ambler G, Sauerbrei $W$. The use of fractional polynomials to model continuous risk variables in epidemiology. Int J Epidemiol 1999:28:964-74.

22 Royston P, Sauerbrei W. Stability of multivariable fractional polynomial models with selection of variables and transformations: a bootstrap investigation. Stat Med 2003;22:639-59.

23 United Nations. World Population Prospects. The 2012 Revision. New York: United Nations, 2014.

24 Souza JP, Cecatti JG, Faundes A, Morais SS, Villar J, Carroli G, et al. Maternal near miss and maternal death in the World Health Organization's 2005 global survey on maternal and perinatal health. Bull World Health Organ 2010;88:113-9. 
25 Greenland S. Ecologic versus individual-level sources of bias in ecologic estimates of contextual health effects. Int I Epidemiol 2001;30:1343-50

26 Wakefield J, Haneuse SJ. Overcoming ecologic bias using the two-phase study design. Am J Epidemiol 2008;167:90816

27 Riskin A, Gonen R, Kugelman A, Maroun E, Ekhilevitch G. Does cesarean section before the scheduled date increase the risk of neonatal morbidity? IMAJ 2014;16:559-63.

28 Souza JP, Gülmezoglu AM, Vogel J, Carroli G, Lumbiganon P, Qureshi Z, et al. Moving beyond essential interventions for reduction of maternal mortality (the WHO Multicountry Survey on Maternal and Newborn Health): a cross-sectional study. Lancet 2013;381: 1747-55.

29 Lurie S, Raz N, Boaz M, Sadan O, Golan A. Comparison of maternal outcomes from primary cesarean section during the second compared with first stage of labor by indication for the operation. Eur J Obstet Gynecol Reprod Biol 2014;182:43-7.

30 Betran AP, Torloni MR, Zhang J, Ye J, Mikolajczyk R, DeneuxTharaux $C$, et al. What is the optimal rate of caesarean section at population level? A systematic review of ecologic studies. Reprod Health 2015:12:57.

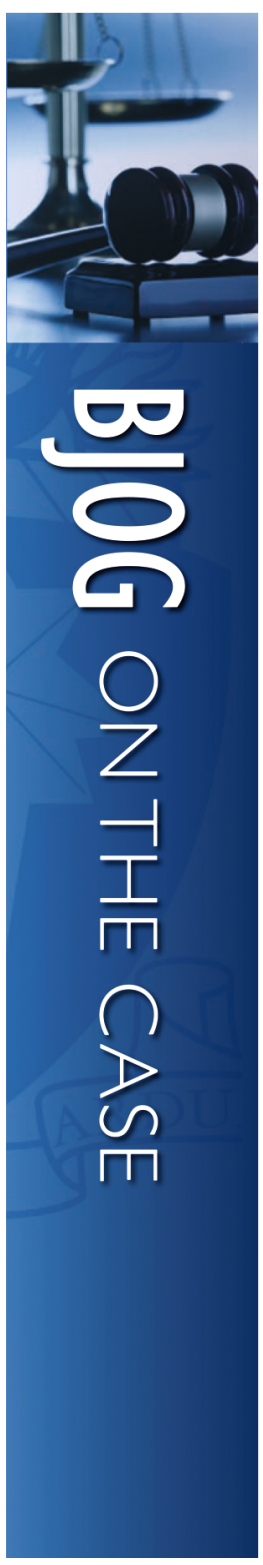

\section{Pain during caesarean section}

With around $92 \%$ of all sections in the UK now carried out under regional (spinal, epidural or combined spinal-epidural) anaestheric general anaesthesia, previously one of the top causes of maternal mortality, have been largely controlled. But in their place has risen the spectre of pain during caesarean delivery, now almost certainly the commonest successful negligence claim against anaesthetists.

Complete comfort cannot be guaranteed for patients being delivered surgically when awake because, although the level of block required for a caesarean section extends to the upper thoracic dermatomes, sometimes this is not enough. We know that a small proportion of patients will feel pain and that in some cases this will warrant intraoperative conversion to general anaesthesia. The Royal College of Anaesthetists sets an audit standard that fewer than $5 \%$ of elective patients should feel pain and that fewer than 1\% should require conversion to general anaesthesia; these figures rise to 20 and $15 \%$ respectively for Category I procedures (http://www.rcoa.ac.uk/document-store/audit-recipe-book-section-8-obstetrics-20 I2).

What would lead to an anaesthetist being regarded as delivering an unacceptably low standard of care in such

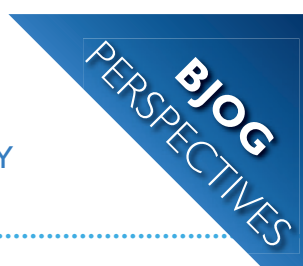
sia, the risks associated with obstet- an instance, a conclusion that, in my own medicolegal casebook, applies to a surprisingly high $74 \%$ of the 76 claims in which I have given an opinion? Problems seem to arise in four main areas.

I During the consent process, it is mandatory to warn patients that they may feel pain which might be severe enough to need converting to general anaesthesia. Without this important information, patients are not able to make a fully informed decision about what anaesthetic technique they prefer. The burden upon doctors in the UK to ensure that patients are provided with sufficient information has been highlighted by the recent Supreme Court verdict in Montgomery: Montgomery v Lanarkshire Health Board (20।5]) UKSC II, (20।5) All ER(D) II3 (Mar).

2 It is essential to test the block carefully, especially the upper level, and ensure that it is high enough before allowing surgery to begin. Cold stimuli such as ethyl chloride are almost universally employed for this purpose, and the patient should not feel this as cold until the fourth thoracic dermatome (T4) is reached, represented by the middle of the breasts. Ideally, even the sensation of touch should be abolished below the T5 level.
3 When the patient complains of pain, it is important to (I) believe her, and (2) take action. This often means temporarily halting surgery (unless the uterus has been incised prior to delivery or haemorrhage control is necessary), giving fast-acting and hopefully effective intravenous analgesia, and ensuring she is comfortable before cautiously continuing.

4 Failure to control pain should lead to an early offer of conversion to general anaesthesia, repeated if initially declined.

Record-keeping is often poor in such cases, making it impossible to confirm that these steps were followed. In addition, many patients seem to be motivated, at least in part, by what they perceive as an uncaring or dismissive attitude on the part of the anaesthetist. The general lessons for any doctor seeking to avoid negligence litigation seem to be much the same no matter what the specialty: keep good show her respect, and be nice. All tors don't get sued (as often).

\section{Disclosure of interests}

Full disclosure of interests available to view online as supporting information.

(c) 2016 Royal College of Obstetricians and records, listen to the patient and other things being equal, nice docGynaecologists 